\title{
ELF and VLF signatures of sprites registered onboard the low altitude satellite DEMETER
}

\author{
J. Błęcki ${ }^{1,3}$, M. Parrot ${ }^{2}$, and R. Wronowski ${ }^{1}$ \\ ${ }^{1}$ Space Research Centre PAS, ul. Bartycka 18a, 00-716 Warsaw, Poland \\ ${ }^{2}$ LPC2E/CNRS, 3A Avenue de la Recherche, 45071 Orléans cedex, France \\ ${ }^{3}$ Collegium Varsoviense, ul. Górczewska 212/226, 01-460 Warsaw, Poland
}

Received: 6 February 2009 - Revised: 19 May 2009 - Accepted: 18 June 2009 - Published: 29 June 2009

\begin{abstract}
We report the observation of ELF and VLF signature of sprites recorded on the low altitude satellite DEMETER during thunderstorm activity. At an altitude of $\sim 700 \mathrm{~km}$, waves observed on the E-field spectrograms at mid-to-low latitudes during night time are mainly dominated by up-going 0+ whistlers. During the night of 20 July 2007 two sprites have been observed around 20:10:08 UT from the observatory located on the top of the mountain Śnieżka in Poland $\left(50^{\circ} 44^{\prime} 09^{\prime \prime} \mathrm{N}, 15^{\circ} 44^{\prime} 21^{\prime \prime} \mathrm{E}, 1603 \mathrm{~m}\right)$ and, ELF and VLF data have been recorded by the satellite at about $1200 \mathrm{~km}$ from the region of thunderstorm activity. During this event, the DEMETER instruments were switched in the burst mode and it was possible to register the wave forms. It is shown that the two sprites have been triggered by two intense $+\mathrm{CG}$ lightning strokes $(100 \mathrm{kA})$ occurring during the same millisecond but not at the same location. Despite the distance DEMETER has recorded at the same time intense and unusual ELF and VLF emissions. It is shown that the whistler wave propagates from the thunderstorm regions in the Earth-ionosphere guide and enters in the ionosphere below the satellite. They last several tens of milliseconds and the intensity of the ELF waveform is close to $1 \mathrm{mV} / \mathrm{m}$. A particularly intense proton whistler is also associated with these emissions.
\end{abstract}

Keywords. Electromagnetics (Guided waves) - Ionosphere (Electric fields and currents) - Meteorology and atmospheric dynamics (Lightning)

Correspondence to: J. Błęcki

(jblecki@cbk.waw.pl)

\section{Introduction}

The purpose of this paper is to report ionospheric ELF/VLF measurement performed onboard the satellite DEMETER when it was at a distance around $1200 \mathrm{~km}$ from a sprite event observed at the top of Śnieżka mountain observatory in Poland. Associated ELF and VLF radiation have been already measured on ground at the time of Transient Luminous Events (TLEs). Bell et al. (1998) have used an ELF/VLF receiving system to measure the magnetic field radiated by positive cloud-to-ground discharges associated with sprites. They found that ELF waveforms have pulses related to intense continuing currents which transfer most of the positive charge to ground during a period of $\sim 1 \mathrm{~ms}$ following the first return stroke. It has been shown by Reising et al. (1999) that the ELF sferic energy is related to the number of sprites produced during a thunderstorm. Ohkubo et al. (2005) have investigated the relationship between the broadband ELF/VLF waveform data and sprite events observed in Japan. They showed that sferic clusters observed in the VLF range are related to the generation of sprites.

DEMETER is devoted to the study of ionospheric perturbations in relation with the seismic activity and the manmade activity. Its payload consists of wave and particle analysers. A large part of the Electromagnetic (EM) waves observed by DEMETER consists of whistlers mainly during night time at low and mid latitudes (Parrot et al., 2008).

Section 2 briefly describes the wave experiment which is a part of the scientific payload of DEMETER, the optical experiment in Śnieżka observatory, and the lightning networks used to characterize the strokes. Specific spectrograms and waveforms with unusual signatures observed by DEMETER are shown in Sect. 3. Section 4 is devoted to the interpretation

Published by Copernicus Publications on behalf of the European Geosciences Union. 


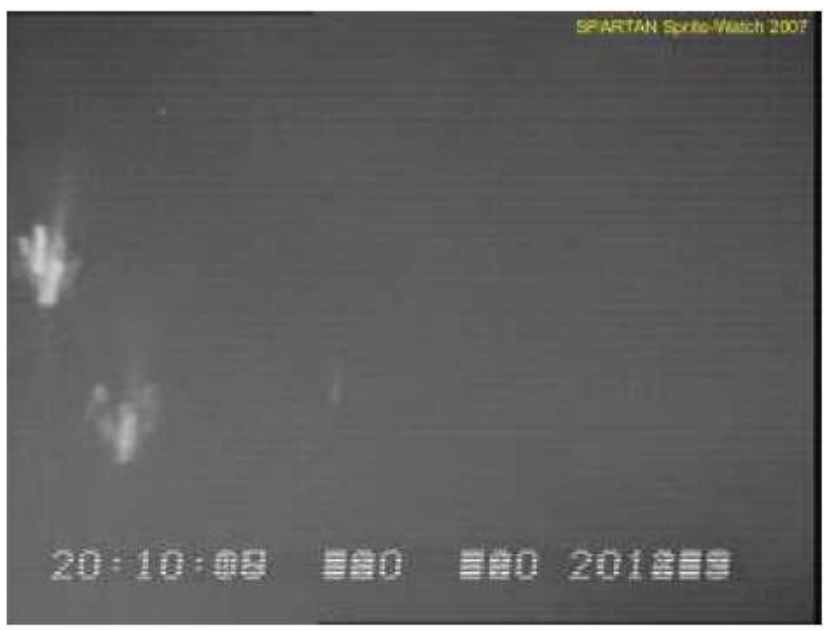

Fig. 1. Picture taken by the camera installed in the observatory at the top of the Śnieżka mountain $\left(50^{\circ} 44^{\prime} 09^{\prime \prime} \mathrm{N}, 15^{\circ} 44^{\prime} 21^{\prime \prime} \mathrm{E}\right.$ $1603 \mathrm{~m}$ ) on 20 July 2007 . Two sprites are clearly seen in this picture. At the time of registration (20:10:08 UT) the camera was oriented in the north-north-west $\left(333^{\circ}\right)$ direction, and the elevation was $30^{\circ}$ (Courtesy A. Odzimek).

of these data manifested in these events, while conclusions are presented in Sect. 5 .

\section{The experiments}

\subsection{Demeter}

DEMETER is a low-altitude satellite $(710 \mathrm{~km})$ launched in June 2004 onto a polar and circular orbit which measures electromagnetic waves all around the Earth except in the auroral zones (Parrot, 2006). In December 2005, the altitude of the satellite was decreased to $660 \mathrm{~km}$. The ELF/VLF range for the electric field is from DC up to $20 \mathrm{kHz}$. There are two scientific modes: a survey mode where spectra of one electric and one magnetic component are onboard computed up to $20 \mathrm{kHz}$ and a burst mode where, in addition to the onboard computed spectra, waveforms of one electric and one magnetic field component are recorded up to $20 \mathrm{kHz}$. The burst mode allows performing a spectral analysis with higher time and frequency resolution. Details of the wave experiment can be found in Parrot et al. (2006) and Berthelier et al. (2006). During the burst mode, the waveforms of the six components of the electromagnetic field are also recorded up to $1.25 \mathrm{kHz}$. This allows performing a detailed wave propagation analysis (Santolik et al., 2006).

\subsection{Optical measurements}

The camera was installed by a Leicester University team in the meteorological observatory at the top of the Śnieżka mountain in south-western part of Poland $\left(50^{\circ} 44^{\prime} 09^{\prime \prime} \mathrm{N}\right.$,

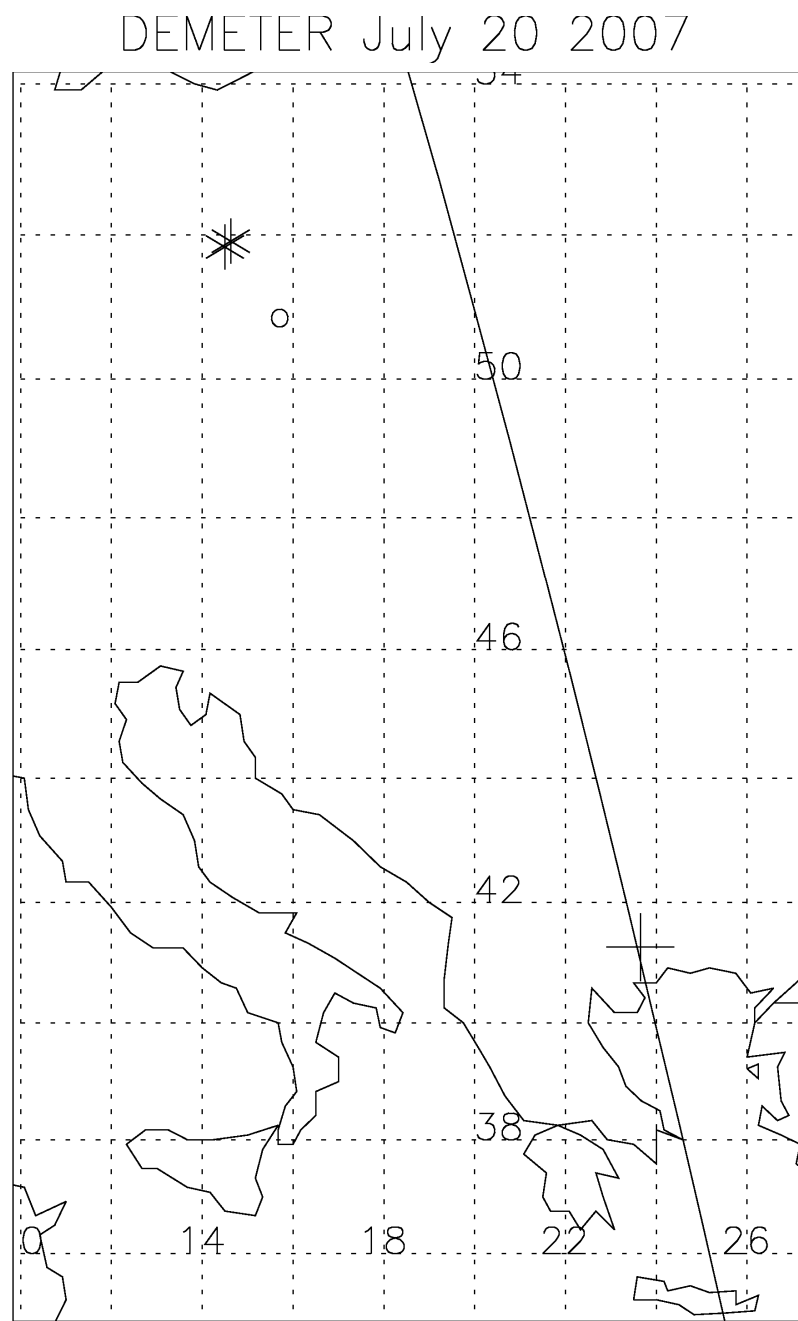

Fig. 2. Map showing the orbit of DEMETER (black line). The sign + indicates the position of DEMETER at the time of the optical observation performed at Śnieżka observatory (the sign o). The two stars give the position of the parent lightning strokes. The distance between the location of the event and the satellite position was around $1200 \mathrm{~km}$.

$15^{\circ} 44^{\prime} 21^{\prime \prime} \mathrm{E}, 1603 \mathrm{~m}$ ) in the frame of the European program SPARTAN Sprite-Watch. For the observations, it was a manually operated system which consists of a monochrome Watec $902 \mathrm{H}$ LLTV camera, a Computar $16 \mathrm{~mm}$ F/1.4 lens, a Pinnacle Dazzle DVD video capture device, a PFD Systems KIWI-OSD video time inserter and a KIWI Garmin GPS receiver, a Manfrotto camera head, a Computar camera housing, and a Sonota Co UFO Capture V2 software installed on a Toshiba Satellite Pro notebook computer. The exposure time of the camera was $40 \mathrm{~ms}$ (A. Odzimek, private communication, 2008). 
Table 1. Parameters of the most intense lightning strokes observed by the EUCLID network close in time and space to the sprites shown in Fig. 1. It must be noticed that L1, L4, L5 and L6 which are eastward are not detected by the LINET network.

\begin{tabular}{ccccc}
\hline Lightning & Day & Time & Position (lat, long) & Current (kA) \\
\hline L1 & 20 Jul 2007 & 20:10:01 885 & 52.35760022 .064000 & -81 \\
L2 & 20 Jul 2007 & $20: 10: 08666$ & 51.44910014 .715100 & 101 \\
L3 & 20 Jul 2007 & $20: 10: 08666$ & 51.37540014 .597900 & 107 \\
L4 & 20 Jul 2007 & $20: 10: 08829$ & 51.74990020 .300600 & -23 \\
L5 & 20 Jul 2007 & $20: 10: 08833$ & 52.46690022 .606200 & -79 \\
L6 & 20 Jul 2007 & $20: 10: 08834$ & 51.06630021 .680100 & -22 \\
L7 & 20 Jul 2007 & 20:10:09 226 & 51.50410014 .427000 & -35 \\
\hline
\end{tabular}

\subsection{The lightning networks}

Two lightning detection networks were used to characterize the lightning strokes in the region of thunderstorm activity: The Munich University lightning detection network named LINET (Betz et al., 2009) and the EUCLID (European Cooperation for Lightning Detection) network. EUCLID provides interconnection of national lightning detecting networks with the aim to identify and detect lightning all over the European area (http://www.euclid.org/).

\section{Observation of the events}

On the night of 20/21 July 2007 during a strong thunderstorm activity two sets of sprites were recorded by the camera from the observatory on the top of the Śnieżka mountain. The first set consisting of two sprites was recorded around 20:10:08 UT, and the second one at 00:18:45 UT. In the following, only the first set of sprites will be considered as the satellite DEMETER was very far at the time of the second event. Figure 1 shows the photo of the sprites recorded around 20:10:08 UT. These sprites were registered when the camera was oriented to the north-north - west directions $\left(333^{\circ}\right)$ with an elevation of $30^{\circ}$. As we can consider that the average altitude of the bottom of the sprites is $55 \mathrm{~km}$ (Lyons, 1996), it means that the sprites were located not farther than $100 \mathrm{~km}$ from the observatory. One can see on the left part of the picture two sprites well developed and an unidentified very weak luminosity in the middle. These two sprites have been simultaneously captured which means that they occur during a time interval shorter than the exposure time of the camera $(40 \mathrm{~ms})$. The thunderstorm cloud system was moving from the territory over Germany to the east-south of Poland and to Czech Republic. Figure 2 shows the orbit of the Demeter satellite (full line), the position of the observatory $(0)$ and the position of the satellite at the time of this event (cross). The registration of the electromagnetic emissions at the time of the observed sprites by DEMETER was done when the satellite was in burst mode with a foot point at about $1200 \mathrm{~km}$.
Figure 3 represents these ELF/VLF data recorded by DEMETER during the event between 20:10:08.274 and 20:10:09.298 UT. Figure 3a shows the wave form of the VLF signal whereas Fig. $3 \mathrm{~b}$ presents the corresponding spectrogram of this wave form. Figure $3 \mathrm{c}$ and d displays the same plots for the ELF range. Many whistlers can be observed in Fig. $3 b$ but two of them are particularly strong as it can be also seen with the waveform shown in Fig. 3a. They start at $\sim 20: 10: 08.666$ and $\sim 20: 10: 08.817$ UT. These two whistlers are also detected in the ELF range (Fig. $3 \mathrm{c}$ and d) with a delay due to the dispersion (20:10:08.703 and 20:10:08.874 UT). This dispersion is induced by wave propagation time which is longer at lower frequencies. They are associated to proton whistlers, the first one being very intense. Looking to the ELF waveform and the intensity of the ELF spectrogram it can be seen that the first whistler is much stronger than the second whereas it is the opposite in the VLF range. The time variation of the waveform of the first whistler (in VLF and in ELF) is not usual as its intensity does not monotonously decrease. In fact the ELF waveform shown in Fig. 3c displays four different wave packets. The second is uncommon and the third can be associated with the proton whistler as it can be seen on the spectrogram in Fig. 3d.

Checking now the lightning occurrence in the zone where the two sprites have been observed with the networks, LINET indicates that there is a strong stroke $(105 \mathrm{kA})$ at 20:10:08.666 UT located at lat $=51.37^{\circ}$ and long $=14.59^{\circ}$. At the same location and at the same time, EUCLID (see Table 1) confirms the presence of this strong stroke (L2 with $107 \mathrm{kA}$ ) but gives another strong stroke (L3 with $101 \mathrm{kA}$ ) at the same time (same ms) but at a slightly different location (lat $=51.45^{\circ}$ and long $=14.71^{\circ}$ ). The distance between these two strokes L2 and L3 is $\sim 12 \mathrm{~km}$. The distance between the observatory and the strokes is $\sim 110 \mathrm{~km}$. Their positions are displayed in Fig. 2 (stars). Both strokes were CG (cloudground) with positive discharge. The whistler which is observed at $\sim 20: 10: 08.817 \mathrm{UT}$ in Fig. 3a must correspond to the lightning stroke L4, L5, or L6 due to uncertainty in the time accuracy of DEMETER which is $10 \mathrm{~ms}$. But considering the intensity of the current it is most probably due to L5. 
(a)
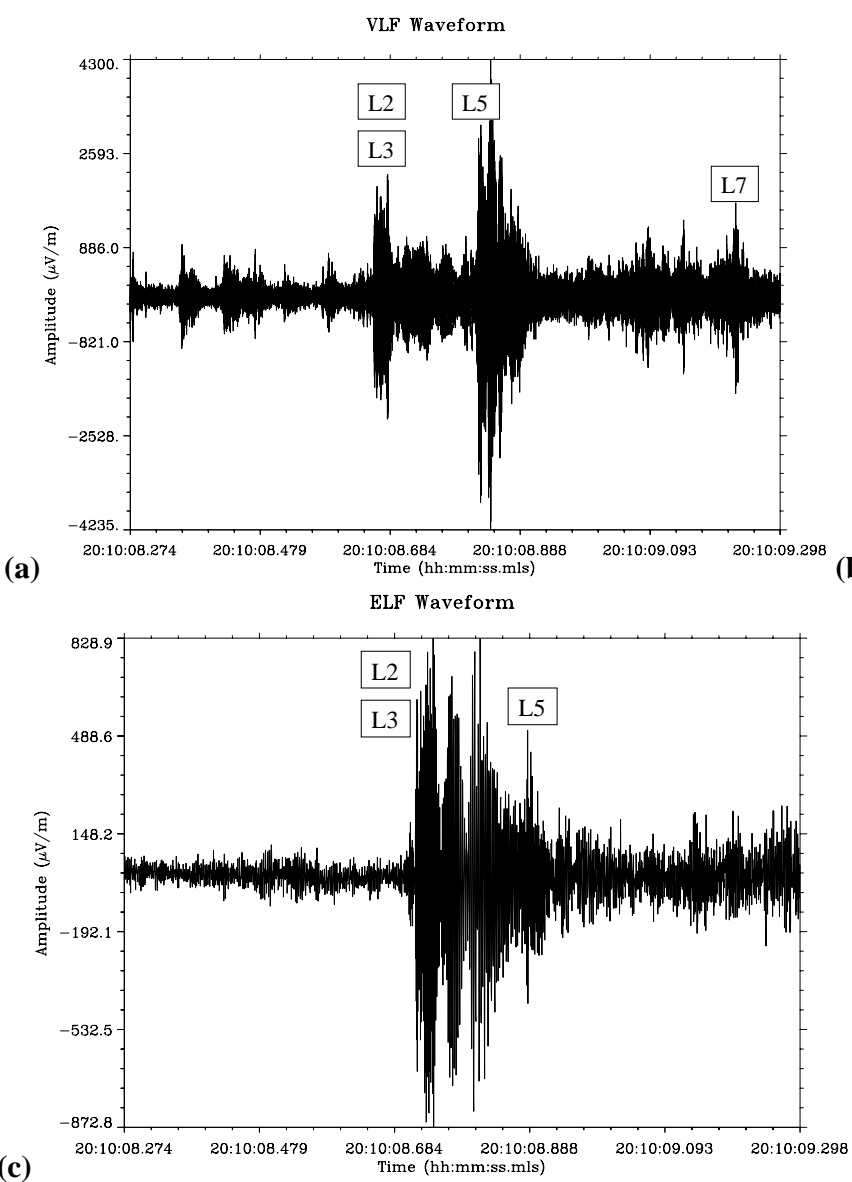

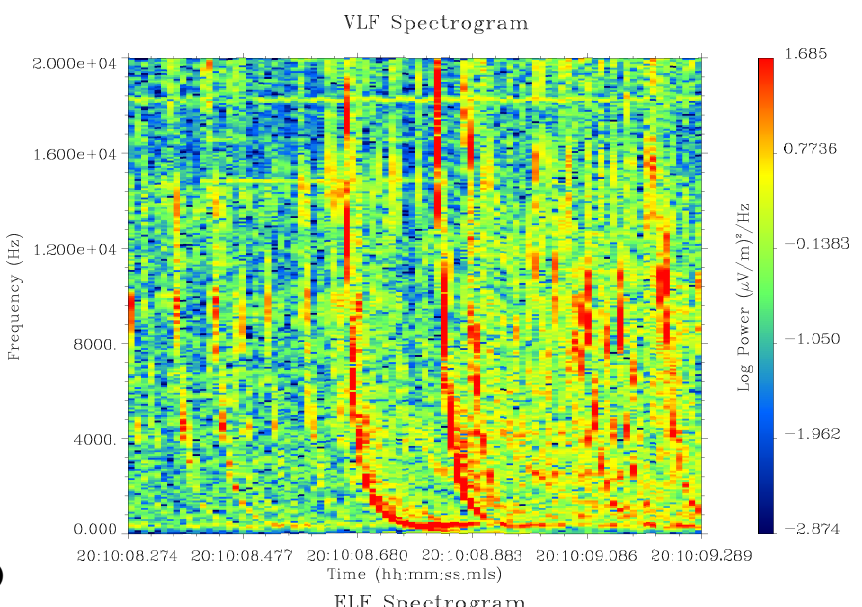

ELF Spectrogram

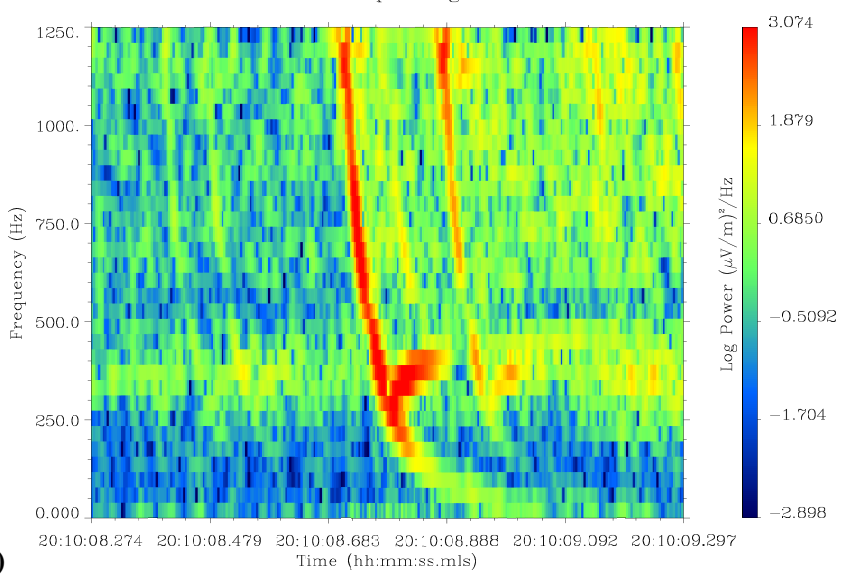

Fig. 3. ELF/VLF data from the electric field experiment onboard the DEMETER satellite gathered during the time of the sprite registration: (a) wave form of the VLF signal, horizontal axis - time (UT), vertical axis - value of the electric field in $\mu \mathrm{V} / \mathrm{m}$, (b) spectrogram of the signal shown in (a), horizontal axis - time, vertical axis - frequency, the intensity is color-coded according to the scale on the right, (c) wave form of the ELF signal, (d) Spectrogram of the ELF signal shown in (c). The discussion is given in the text.

The fact that one can distinguish three peaks in the waveform cannot be attributed to the three lightning strokes L4, L5, L6 because their time of occurrence is too close. It is most probably due to the whistler wave of L5 reaching the spacecraft after slightly different propagation paths. The last wave packet in Fig. 3a must correspond to L7 in Table 1.

\section{Analysis of the event}

During the time interval of interest there is no other strong lightning strokes recorded by the networks. Despite the fact that the photo of the sprites is localized in time with an accuracy of only one second (20:10:08 UT), it is therefore possible from lightning networks to determine more precisely the time when the sprites must occur. Considering the accuracy of the time onboard DEMETER and the propagation time of the wave between the location of the strokes and the satellite which must not exceed $10 \mathrm{~ms}$, it could be able to correlate the waveform as well as the spectrogram of the ELF/VLF signal shown in Fig. 3 with the time of the thunderstorm discharges shown in Table 1. The low altitude satellite DEMETER is able to catch whistler signatures at such distance $(1200 \mathrm{~km})$ as it was shown by Fiser et al. (2009). Then, there is no doubt that the strong lightning observed on ground at 20:10:08.666 UT and onboard the satellite at the same time has triggered the sprites observed in Fig. 1. The point is that there are two lightning strokes detected by the network EUCLID at 20:10:08.666 UT during the same millisecond and that two sprites are simultaneously observed. The time delay between the two sprites may be $40 \mathrm{~ms}$ but the lightning networks indicate that there is no strong stroke able to trigger a sprite between 20:10:08.666 and 20:10:08.706 UT in this area. Could it be possible that each lightning triggers a sprite? Although it is known that sprites could occur at a distance up to $50 \mathrm{~km}$ from the parent lightning (Lyons, 1996; Wescott et al., 2001), the geometry of the observations indicates that the two sprites occur close to the positions of 
DEMETER 2007-07-20 20:10:06.993 - 2007-07-20 20:10:11.024

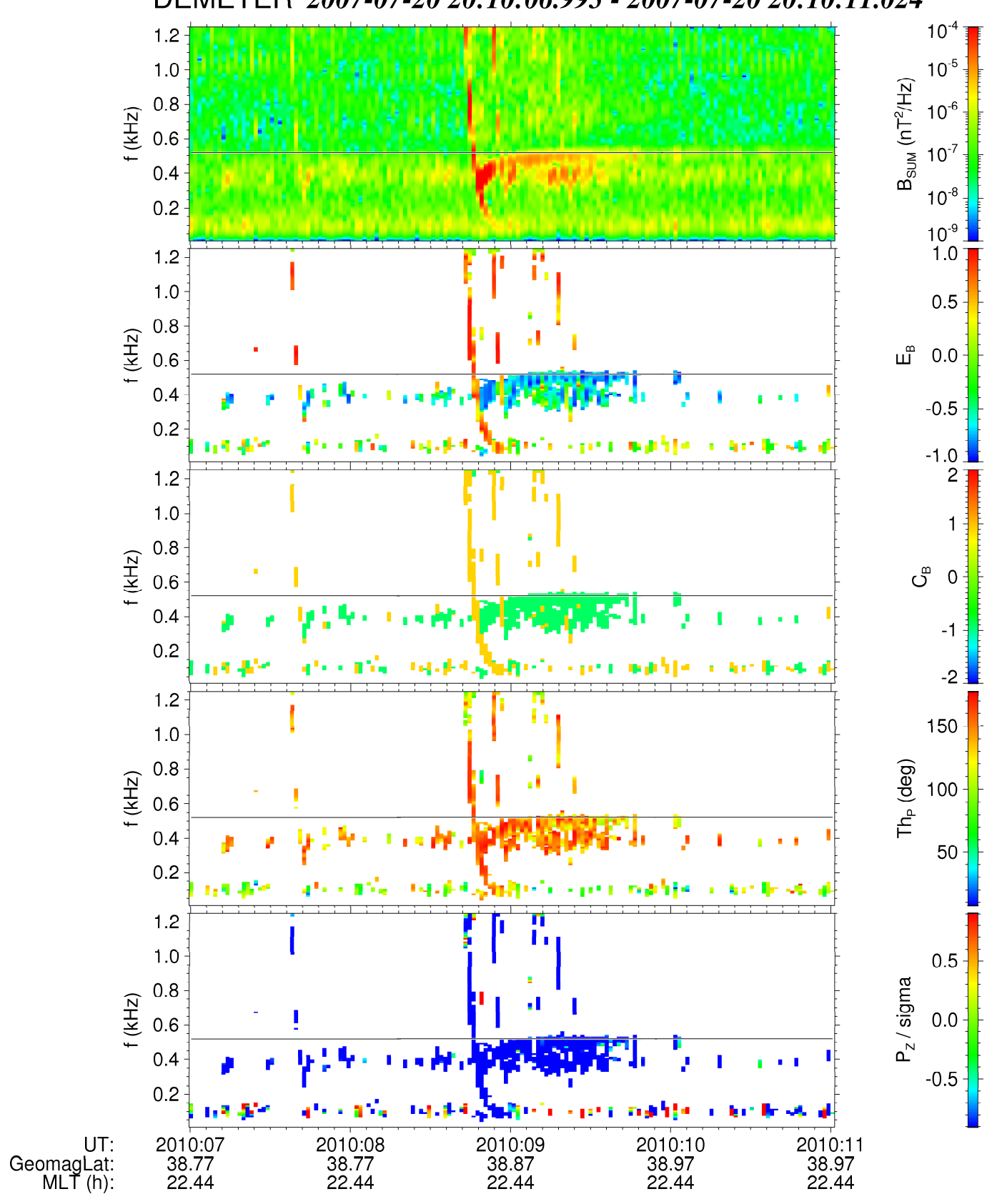

Fig. 4. Analysis of the propagation of the ELF signal during the event as function of the time. From the top to the bottom, the first panel shows the spectrum of the magnetic field variations (sum of the 3 components), the second panel displays the ellipticity of magnetic field polarization - value of 1 means right-hand circularly polarized waves, linear polarization gives 0 , and -1 means left-hand circular polarization, the third panel is the sense of polarisation of the waves, the fourth panel indicates the angle between the wave vector and the Earth's magnetic field calculated by the SVD technique (Santolik et al., 2003), and the bottom panel represents the Poynting vector. Empty areas in the four bottom panels correspond to magnetic power-spectral densities below $10^{-6} \mathrm{nT}^{2} \mathrm{~Hz}^{-1}$. The geomagnetic latitude and the Magnetic Local Time (MLT) are displayed at the bottom of the figure. The discussion of the results is given in the text.

the two lightning strokes. It has been already shown that the sprites and the lightning are at nearly the same distance from the observatory $(100-110 \mathrm{~km})$ and in the same direction. If we consider that the altitude extension of a sprite is, in average, of the order of $50 \mathrm{~km}$, Fig. 1 indicates that the distance between these two sprites is close to $10 \mathrm{~km}$ which is approximately the distance between the two strokes. Therefore there is a large probability that both lightning strokes L2 and L3 trigger a sprite. 




Fig. 5. VLF wave form from the electric field experiment onboard the DEMETER satellite gathered at the time of L1 (see Table 1).

Coming back to Fig. 3c, it is shown that the first ELF wave packet which corresponds to the two lightning strokes has very high maximum amplitude close to $1 \mathrm{mV} / \mathrm{m}$. It is followed by another wave packet which can be attributed to the sprites and which is similar to broadband sferic cluster observed on ground at Nançay (France) by Van der Velde et al. (2006). This wave packet is also similar to the secondary ELF perturbations observed by Ohkubo et al. (2005). The third ELF wave packet is related to the strong proton whistler which is associated to the parent lightning L2 and L3 of the sprites. The fourth one can be attributed to L5. Figure 4 gives the results of the analysis of the ELF signal propagation. The upper panel presents the spectrum of the magnetic field fluctuations. The next two panels give the information on the polarization of the waves which is in agreement with polarization of the electron whistlers. As usual, the ion whistlers have opposite polarization as it is seen in the diagram. The next two panels show the direction of the propagation. The fourth panel gives the angle between the magnetic field and the wave vector. We see that this angle is close to the $180^{\circ}$ which means that the wave propagation is opposite to the magnetic field direction and for the North Hemisphere that the wave is coming from a region below satellite. This information is confirmed in the bottom panel where the Poynting vector is presented. Its negative value also indicates that the origin of the waves is below the satellite orbit. It means that the lightning wave first propagates in the Earth-ionosphere guide and then enters in the ionosphere in a region below the satellite. In order to compare with another whistler recorded by DEMETER but without an associated sprite, Fig. 5 shows the VLF waveforms associated with L1 in Table 1. This lightning stroke occurs a few seconds before L2 and L3 in a location not very far from L2 and L3. One can observe a classical decrease of the amplitude of the waveforms in VLF range which is very different from the variations shown in Fig. 3a for L2 and L3.

\section{Conclusions}

The observations discussed in this paper are one of the first in situ registrations of the electromagnetic effects of the sprites in the ionosphere. In spite of the quite distant position of the satellite Demeter relative to the events the recorded effects are clearly distinguished and strong. The two sprites observed on ground by the camera installed on the top of the mountain Śnieżka are associated with intense thunderstorm activity. It has been shown that these two sprites can be attributed to two +CG lightning strokes registered by the network EUCLID. These two strokes have large current intensity of the order of $100 \mathrm{kA}$. But the available information were not enough to estimate the charge moment change although it is important to characterize the parent lightning of sprites. At the same time, Demeter registered very intense emissions in VLF and ELF range (whistlers and proton whistlers). It is shown that these emissions are different from the ones recorded at the time of classical whistlers because they do not monotonously decrease and they last several tens of milliseconds after the occurrence of the parent lightning. Due to this timing, the persistence of wave activity is attributed to the electrostatic discharge in the sprite. This is particularly true for the ELF waves. In the past, it has been shown (Bell et al., 1998; Reising et al., 1999) that strong ELF sferic energy could be an indicator of a sprite. Cummer et al. (1998) have shown that currents associated to sprite exhibit a second peak simultaneous in time with sprite luminosity. They suggest that the ELF radiation is produced by this current. For this event, the ELF wave intensity is close to $1 \mathrm{mV} / \mathrm{m}$ and an intense proton whistler is observed.

Acknowledgements. This work was supported by the Centre National d'Etudes Spatiales (for MP), and by the grant MNiSW N $\mathrm{N} 307065834$ (for JB and RW). It is based on observations with the electric field experiment ICE and the magnetic field experiment IMSC embarked on DEMETER. The authors thank J. J. Berthelier the PI of the electric field experiment for the use of the data and Anna Odzimek for discussion and photo of sprites. We also thank H. D. Betz (LINET) and G. Diendorfer (EUCLID) for the information on the thunderstorm activity. The authors acknowledge support from the grant CNRS-PAN no. 15209.

Topical Editor M. Pinnock thanks two anonymous referees for their help in evaluating this paper.

\section{References}

Bell, T. F., Reising, S. C., and Inan, U. S.: Intense continuing currents following positive cloud-to-ground lightning associated with red sprites, Geophys. Res. Lett., 25(8), 1285-1288, 1998.

Berthelier, J. J., Godefroy, M., Leblanc, F., Malingre, M., et al.: ICE, the electric field experiment on DEMETER, Planet. Space Sci., 54, 456-471, doi:10.1016/j.pss.2005.10.016, 2006.

Betz, H. D., Schmidt, K., Laroche, P., Blanchet, P., Oettinger, W. P., Defer, E., Dziewit, Z., and Konarski, J.: LINET - An international lightning detection network in Europe, Atmos. Res., 91, 564-573, doi:10.1016/j.atmosres.2008.06.012, 2009. 
Cummer, S. A., Inan, U. S., Bell, T. F., and Barrington-Leigh, C. P.: ELF radiation produced by electrical currents in sprites, Geophys. Res. Lett., 25, 1281-1284, 1998.

Fiser, J., Chum, J., Diendorfer, G., Parrot, M., and Santolik, O.: Whistler intensities above thunderstorms, Ann. Geophys., submitted, 2009.

Lyons, W. A.: Sprite observations above the U.S. High Plains in relation to their parent thunderstorm systems, J. Geophys. Res., 101(D23), 29641-29652, 1996.

Ohkubo, A., Fukunishi, H., Takahashi, Y., and Adachi, T.: VLF/ELF sferic evidence for in-cloud discharge activity producing sprites, Geophys. Res. Lett., 32, L04812, doi:10.1029/2004GL021943, 2005.

Parrot, M. (Ed.): First results of the DEMETER micro-satellite, Special Issue of Planet. Space Sci., 54(5), 2006.

Parrot, M., Benoist, D., Berthelier, J. J., Blecki, J., et al.: The magnetic field experiment IMSC and its data processing onboard DEMETER: Scientific objectives, description and first results, Planet. Space Sci., 54, 441-455, doi:10.1016/j.pss.2005.10.015, 2006.
Parrot, M., Berthelier, J. J., Lebreton, J. P., Treumann, R., and Rauch, J. L.: DEMETER observations of EM emissions related to thunderstorms, Space Sci. Rev., 137, 511519, doi:10.1007/s11214-008-9347-y, 2008.

Reising, S. C., Inan, U. S., and Bell, T. F.: ELF sferic energy as a proxy indicator for sprite occurrence, Geophys. Res. Lett., 26(7), 987-990, 1999.

Santolík, O., Parrot, M., and Lefeuvre, F.: Singular value decomposition methods for wave propagation analysis, Radio Sci., 38(1), 1010, doi:10.1029/2000RS002523, 2003.

Santolik, O., Nemec, F., Parrot, M., Lagoutte, D., Madrias, L., and Berthelier, J. J.: Analysis methods for multi-component wave measurements on board the DEMETER spacecraft, Planet. Space Sci., 54, 512-527, 2006.

Van der Velde, O. A., Mika, A., Soula, S., Haldoupis, C., Neubert, T., and Inan, U. S.: Observations of the relationship between sprite morphology and in-cloud lightning processes, J. Geophys. Res., 111, D15203, doi:10.1029/2005JD006879, 2006.

Wescott, E. M., Stenbaek-Nielsen, H. C., Sentman, D. D., Heavner, M. J., Moudry, D. R., and Sabbas, F. T. S.: Triangulation of sprites, associated halos and their possible relation to causative lightning and micrometeors, J. Geophys. Res., 106(A6), 1046710477, 2001. 